\title{
Screening strategy for gestational diabetes mellitus in a rural health setting in Kanpur (UP)
}

\author{
Ashok Kumar Maini ${ }^{1, *}$, S.P. Singh ${ }^{2}$, Ajay Bhagoliwal ${ }^{3}$, Amrita $^{4}$ \\ ${ }^{\mathbf{1 , 2}}$ Assistant Professor, ${ }^{3}$ Professor Bio-Statistic, ${ }^{4}$ MBBS Student, Rama Medical College Mandhana Kanpur, Uttar Pradesh, India
}

*Corresponding Author:

Email: ashokanitamaini@gmail.com

\begin{abstract}
Introduction: Gestational Diabetes Mellitus (GDM) is defined as carbohydrate intolerance with its onset or diagnosis in pregnancy. Changing lifestyle and food habits are largely responsible for increasing incidence of Diabetes Mellitus, especially, in younger age groups. The same increasing trend is being observed in gestational diabetes mellitus. Treatment of GDM improves perinatal as well as maternal outcome. Timely diagnosis of GDM may result in reduced maternal and neonatal morbidity. The high risk pregnancies due to GDM contribute highly towards poor maternal and fetal outcome. GDM is one of the major causes for increased incidence of Macrosomia and primary Cesarean section abnormal 75-g GTT results show strong correlation with macrosomia.

Materials and Methods: The sample size was calculated using the formula for comparing the means of two groups, using the results of Boyd, R. Leigh, B. and Stuart, P. as preliminary estimate for the average difference in mean capillary and venous blood sugar. A sample of 60 ANC cases has been studied as per inclusion and exclusion criteria.

Aim and Objectives: Improvement in the diagnostic ability of the screening test for Diabetes Mellitus by comparing the commonly used method of using RBS with fasting $2 \mathrm{hr}$ GTT.

Result: The mean age was found to be $27.75 \pm 4.31$ years. Mean Gestational age of the cases was $27.53 \pm 7.29$ Weeks. Mean OGTT (fasting) has been found to be statistically significantly higher than their RBS (' $t$ ' $=5.731 \mathrm{p}<0.001$ ). GDM was diagnosed in 5\% cases when RBS was used as diagnostic criteria whereas fasting OGTT criteria classified 20\% cases as GDM in the same sample, this difference is statistically highly significant.

Conclusion: A new strategy for using fasting OGTT as screening test has amongst ANC cases of rural population been suggested.
\end{abstract}

Keywords: Screening, GDM, RBS, 2 hour OGTT.

\section{Introduction}

Gestational diabetes mellitus (GDM) is defined as carbohydrate intolerance with its onset or diagnosis in pregnancy. ${ }^{1}$ Changing lifestyle and food habits are largely responsible for increasing incidence of Diabetes Mellitus, especially, in younger age groups. The same increasing trend is being observed in gestational diabetes mellitus. ${ }^{2,3}$ Treatment of GDM improves perinatal as well as maternal outcome. ${ }^{1}$ Timely diagnosis of GDM may result in reduced maternal and neonatal and perinatal morbidity. The high risk pregnancies due to GDM contribute highly towards poor maternal and fetal outcome. GDM is one of the major causes for increased incidence of Macrosomia and primary Cesarean section ${ }^{4,5}$ Abnormal 75-g GTT results show strong correlation with macrosomia, ${ }^{6}$ high cesarean section rates, ${ }^{4}$ and poor perinatal outcomes (higher the two-hour post-intake glucose levels, the poorer the outcome). ${ }^{4,5}$ The aim of this study is to evaluate GTT as a diagnostic tool as compared to random blood sugar (RBS).The current practice amongst medical persons dealing with ANC cases in primary health care system in India is to use RBS to identify and rule out GDM. The Diabetes in Pregnancy Study Group of India (DIPSI) guidelines recommend the non-fasting glucose tolerance test as a single step screening and diagnostic test for gestational diabetes mellitus (GDM). The World Health Organization
(WHO) $1999^{3}$ recommends fasting oral glucose tolerance test (Fasting OGTT) as a screening test for GDM. Studies have also reported higher incidence of pre-eclampsia, clinical neo-natal hypoglycemia and hyperbilirubinemia amongst women with GDM as compared to normal women. It is therefore desirable to diagnose GDM in early pregnancy for proper management and improved prognosis. Therefore it is essential to evaluate the efficacy of fasting OGTT as compared to RBS as a screening test for GDM. Further, a very large majority of the population of India lives in rural areas, having little access to advanced medical facilities. Blood sugar estimation by glucometer can be an effective alternative. R Boyd, B Leigh and P Stuart found that the mean laboratory blood glucose was 7.075 $\mathrm{mmol} / \mathrm{l}$, and the mean capillary blood glucose was 7.66 $\mathrm{mmol} / \mathrm{l}$, giving a statistically significant difference $(p=0.001)$ between the mean values for the laboratory and capillary glucose samples $\left(0.58 \mathrm{mmol} / \mathrm{l} .{ }^{7}\right.$ But this difference is clinically not significant, at least in the mid ranges. In case of extreme outlier values, Laboratory investigation methods should be relied upon. Therefore we decided $\mu$ to compare RBS results with fasting OGTT with 75 gram glucose on a randomly chosen ANC cases attending Rama Medical College, Hospital \& Research Centre. Glucometer along with pre prepared packets of $75 \mathrm{gm}$ glucose can 
be distributed to Asha Bahu, and other health care workers in rural areas. Thus the results of this study may have a very important role in a community setting.

\section{Aim and Objectives}

Aim Early and reliable diagnosis of GDM.

Objective Compare the diagnostic ability of RBS and 2 hour OGTT in diagnosing GDM

\section{Materials and Methods}

The sample size was calculated using the formula for comparing the means of two groups, using the results of Boyd, R. Leigh, B. and Stuart, P. ${ }^{7}$ As preliminary estimate for the average difference in mean capillary and venous blood sugar

$$
\left.\mathrm{N} \geq \frac{\left.\left(Z_{1-\alpha / 2}\right)+Z_{1-\beta}\right)^{2}\left(\sigma_{1}^{2}+\sigma_{2}^{2}\right)}{\left(\mu_{1}-\mu_{2}\right)^{2}}=(7.84 * 1.98) / 0.34=45.65\right)
$$

Therefore we decided to have a sample size of 60 ANC cases selected from the field attached to the Rural Health Training Centre (RHTC) of the Community Medicine department of Rama Medical College, Kanpur. Subjects were selected using simple random sampling technique from the ante natal cases attending the OPD of RHTC. For this a list of 60 random numbers between 1 to 150 was generated. ANC bearing the serial number corresponding to the random numbers generated as above were selected from the A N C register. If the selected case did not meet the inclusion criterion then next case was included. The process was repeated till the desired sample size was achieved.

Ethical Consideration: Prior approval was obtained from the Institutional medical ethics committee.

Inclusion Criterion: All antenatal cases (II \& III trimester) attending the ante natal clinic of the Rural Health Training Center (RHTC) of the Rama Hospital \& Research Centre.

\section{Exclusion Criterion}

1. All cases previously diagnosed as diabetic or hypertensive.

2. Those cases who did not give their consent for GTT.

3. Cases suffering from any co morbidity like hypo/hyperthyroidism.

The blood sugar estimation was done using a glucometer with uncorded strips. Random blood sugar was done when the case came for checkup at RHTC. For fasting OGTT, the case was advised to come next day nil orally next morning at 8 A.M. After doing fasting blood sugar, the case was administered 75 gram glucose orally and blood sugar estimation was done after 1 hour and 2 hours. The norms given by ADA diabetes care Association 2003. ${ }^{6}$ Were followed.

\section{Results}

Table 1: Gestational age wise distribution of the cases

\begin{tabular}{|l|c|c|}
\hline $\begin{array}{c}\text { Gestational Age (in } \\
\text { Weeks) }\end{array}$ & No. & Percentage \\
\hline $12-20$ & 12 & 20.0 \\
\hline $20-30$ & 22 & 36.7 \\
\hline$>30$ & 26 & 43.3 \\
\hline \multicolumn{2}{|c|}{ Mean G.A. $=\mathbf{2 7 . 5 3} \pm \mathbf{7 . 2 9}$ Weeks } \\
\hline
\end{tabular}

The Maximum cases $(43.3 \%)$ were of gestational age more than 30 . Mean gestational age of our sample is $27.53 \pm 7.29$ weeks.

Table 2: Age wise distribution of the cases

\begin{tabular}{|l|c|c|}
\hline \multicolumn{1}{|c|}{ Age (in years) } & No. & \% \\
\hline $20-25$ & 12 & 20 \\
\hline $25-30$ & 26 & 43.4 \\
\hline $30-35$ & 14 & 23.3 \\
\hline$>35$ & 8 & 13.3 \\
\hline Total & $\mathbf{6 0}$ & $\mathbf{1 0 0}$ \\
\hline Mean Age $=\mathbf{2 7 . 7 5} \pm \mathbf{4 . 3 1}$ \\
\hline
\end{tabular}

Table 2: shows that maximum cases belonged to age group $25-30$ with mean age $27.75 \pm 4.31$.

Table 3: Showing mean blood sugar levels

\begin{tabular}{|l|c|}
\hline \multicolumn{1}{|c|}{ Blood Sugar levels } & Mean \pm S.D. \\
\hline fasting & $88.5 \pm 8.79$ \\
\hline OGTT (2 Hour) F & $139.03 \pm 23.88$ \\
\hline R.B.S. & $116.7 \pm 18.46$ \\
\hline 't' OGTT (2 Hour) Vs. RBS $=\mathbf{5 . 7 3 1}$ p $<\mathbf{0 . 0 0 1}$ \\
\hline
\end{tabular}

The mean blood sugar levels found on 2 hour OGTT is $139.03 \pm 23.88$ which is much higher than RBS levels (116.7 \pm 18.46$)$ done in the same cases.

Table 4: Showing cases detected as GDM by RBS and Fasting OGTT (2hours) (N=60)

\begin{tabular}{|c|c|}
\hline $\begin{array}{c}\text { No. of cases having B.S. }> \\
\mathbf{1 5 5} \text { after } \mathbf{2} \text { hours }\end{array}$ & $\begin{array}{c}\text { No. of cases having } \\
\text { B.S. }>\mathbf{1 4 0} \text { (RBS) }\end{array}$ \\
\hline $12(20 \%)$ & $3(5 \%)$ \\
\hline $\mathbf{Z}=\mathbf{2 . 4 2} ; \mathbf{p}=\mathbf{0 . 0 1 3}$ & \\
\hline
\end{tabular}

Thus the missed cases of GDM by RBS $=15 \%(95 \%$ CI $13.4 \%-18.7 \%$ ) as is evident from the above table.

\section{Discussion}

The mean age of our sample was $27.75 \pm 4.31$ which is higher than reported by Farrar D, Duley L and Lawlor D A 2017 (24.1 $27.75 \pm 3.1) .{ }^{9}$ Mean gestational age has been found to be $27.53 \pm 7.29$ Weeks as compared to $23.7 \pm 7.6$ found by Farrar, D et al. ${ }^{9}$

Table 3 shows the mean blood sugar levels of fasting, and 2 hour samples. Mean RBS blood sugar the number of pregnancy induced diabetes cases in rural areas is growing fast. Screening for GDM is very 
important in order to improve feto-maternal outcome. It is therefore necessary to identify a reliable screening technique, which may be used in rural setting also.

Levels are significantly lower than the mean OGTT ( 2 hours) levels in the same cases. This is a important indicator that detection of GDM should not be based on RBS.

Table 4 that $20 \%$ cases were classified as GDM using the 2 hour fasting OGTT criterion whereas only $5 \%$ case could be classified as GDM when RBS was used. This difference is statistically significant $(Z=$ $2.42 \mathrm{p}=0.013)$. Therefore we are missing $15 \%(95 \%$ CI $13.4 \%$ to $18.7 \%$ ) cases of GDM if we use RBS as the diagnostic criterion. In rural areas venous blood sugar is a difficult preposition. Similar results have been reported by Berg, S.A A. et al. ${ }^{8}$

\section{Conclusion}

We propose a strategy to train paramedics posted in rural areas to be equipped with a non-coded Glucometer along with pre-weighed $75 \mathrm{Gm}$. glucose packets. Then train the paramedics posted in rural field. This can go a long way in detecting GDM in its early stages. This will reduce both maternal morbidity and Neo-natal mortality.

\section{References}

1. Ferrara A. Increasing prevalence of gestational diabetes mellitus: a public health perspective. Diabetes Care. 2007;30 Suppl 2.:S141-146. Pmid:17596462

2. Casey BM, Lucas MI, Mcintire DD, Liezemo KJ. Pregnancy outcomes in women with GDM compared with the genetic obstetric population. Obstet Gynecol. 1997;90:869-73.

3. Deerochanawong C, Putiyanun C, Wongsuryrat M, Serirat S, Jinayon P. Diabetes Data Group and World Health Organization criteria for detecting gestational. Comparison of National diabetes mellitus. Diabetologia. 1996;39(9):1070-73.

4. Khan KS, Syed AH, Hashmi FA, Rizvi JH. Relationship of fetal macrosomia to a $75 \mathrm{~g}$ glucose challenge test in nondiabetic pregnant women. Aust N Z J Obstet Gynaecol. 1994;34(1):24-7.

5. Metzger BE, Lowe LP, Dyer AR, Trimble ER, Chaovarindr U. Hyperglycemia and adverse pregnancy outcomes, HAPO Study Cooperative Research Group. $N$ Engl J Med. 2008;358:1991.

6. Deerochanawong C, Putiyanun C, Wongsuryrat M, Serirat S, Jinayon P. Comparison of National Diabetes Data Group and World Health Organization criteria for detecting gestational diabetes mellitus. Diabetologia. 1996;39(9):1070-3.

7. Boyd R, Leigh B, Stuart P. Capillary versus venous bedside blood glucose estimations. Emerg Med J. 2005;22(3):177-9.

8. Berg, S. A.A. Pregnancy diabetes: A comparison of diagnostic protocols based on point-of-care, routine and optimized laboratory conditions. Sci. Rep.5, 16302; doi: 10.1038/srep16302 (2015).

9. Farrar D, Duley L, Dowswell T, Lawlor DA. Different strategies for diagnosing gestational diabetes to improve maternal and infant health. Cochrane Database Syst Rev. 2017;8:CD007122. doi:10.1002/14651858.CD007122.pub4. 